\title{
Teores plasmáticos de hormônios, produção e composição do leite em sala de espera climatizada ${ }^{1}$
}

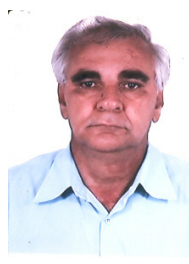

Irineu Arcaro Junior ${ }^{2}$, Juliana R. P. Arcaro ${ }^{2}$, Cláudia R. Pozzi ${ }^{3}$, Helena Fagundes ${ }^{4}$, Soraia V. Matarazzo ${ }^{5}$ \& Claudio A. de Oliveira ${ }^{6}$

\author{
1 Projeto financiado pela FAPESP \\ ${ }^{2}$ CAPTA de Bovinos de Leite, Instituto de Zootecnia. CEP 13460-000, Nova Odessa, SP. E-mail: iarcaro@izsp.br (Foto) \\ ${ }^{3}$ CAPTA de Bovinos de Leite, Instituto de Zootecnia \\ ${ }^{4}$ Bolsista FAPESP \\ ${ }^{5}$ Doutoranda em Física do Ambiente Agrícola - NUPEA, ESALQ/USP \\ ${ }^{6}$ Prof. Dr., Departamento de Reprodução Animal da FMVZ - USP
}

Protocolo $37-2 / 4 / 2002$ - Aprovado em 18/6/2003

\begin{abstract}
Resumo: Através do presente trabalho, avaliou-se a eficiência da climatização do ambiente da sala de ordenha (ventilação ou ventilação mais aspersão) sobre a produção e composição do leite (gordura, proteína, lactose) e os níveis plasmáticos de cortisol, triiodotironina $\left(\mathrm{T}_{3}\right)$ e tiroxina $\left(T_{4}\right)$ Foram utilizadas 21 vacas em lactação, com produção média de $21 \mathrm{~kg} \mathrm{~d}^{-1}$ de leite, distribuídas em um delineamento inteiramente casualisado. O período experimental foi de 90 dias, entre os meses de agosto e outubro. Os tratamentos utilizados foram: 1) sala de espera sem climatização - controle (CONTR); 2) sala de espera com ventilação artificial (V) e 3) sala de espera com ventilação artificial mais aspersão (VA). Os dados referentes à produção de leite foram coletados diariamente em cada ordenha e, para análise da composição do leite, coletaramse amostras semanais. Para a determinação dos hormônios - cortisol, $T_{3}$ e $T_{4}$, foram colhidas amostras de sangue, semanalmente, da veia coccídea. Mediante análises dos dados (ANOVA), verificou-se que não houve diferença entre os tratamentos para a produção de leite, que os animais do tratamento $\mathrm{V}$ apresentaram maior teor de gordura no leite e, ainda, que os animais do grupo CONTR apresentaram maiores concentrações hormonais de cortisol, $T_{3}$ e $T_{4}$, seguida pelo tratamento VA. Por fim, o tratamento VA proporcionou melhores condições de conforto aos animais, porém o tempo de permanência dos animais na sala de ordenha climatizada (30 min) foi, provavelmente, insuficiente para proporcionar aumento na produção de leite.
\end{abstract}

Palavras-chave: estresse térmico, climatização, produção de leite, cortisol, tiroxina, triiodotironina

\section{Plasmatic levels of hormones, production and composition of milk in an acclamatized waiting room}

\begin{abstract}
Absctract: The objective of this study was to evaluate the effects of acclimatization of the milking ambient (ventilation and ventilation + water spraying) on milk production, milk composition (fat, protein, lactose) and plasma concentration of cortisol, $\mathrm{T}_{3}$ and $\mathrm{T}_{4}$. The experiment was conducted from August to October during 90 days. Twenty-one lactating dairy cows with milk yield around $21 \mathrm{~kg} \mathrm{~d}^{-1}$ were allocated three random treatments. The tested treatments were: 1) waiting room without climatization (CONTR); 2) waiting room with ventilation (V) and 3) waiting room with ventilation + water spraying (VA). Milk production data were collected daily and for milk composition, samples were collected once a week. Blood samples for hormone determination were collected weekly, from the coccigea vein. Data were analyzed by computer program SAS PROC ANOVA. Results showed no significant differences among treatments for milk production; the treatment ventilation had higher fat content; and the control group had higher concentrations of cortisol, $\mathrm{T}_{3}$ and $\mathrm{T}_{4}$ followed by treatments 2 and 3 . The treatment VA although increased animal comfort it was insufficient to improve milk production, probably because of short time exposure (30 $\mathrm{min})$.
\end{abstract}

Key words: heat stress, climatization, cortisol, tiroxina, triiodotironina, milk production

\section{INTRODUÇÃO}

As vacas em lactação de alta produção são particularmente sensíveis ao estresse térmico, devido à função produtiva especializada e alta eficiência na utilização dos alimentos. Esses animais apresentam metabolismo mais acelerado, com maior produção de calor endógeno sendo, então, mais susceptíveis às ações do meio ambiente. 
O Brasil possui cerca de dois terços de seu território situados na faixa tropical do planeta, onde predominam altas temperaturas do ar, em virtude da elevada radiação solar incidente. A temperatura média do ar situa-se acima dos $20^{\circ} \mathrm{C}$, sendo que a temperatura máxima se apresenta, nas horas mais quentes do dia, acima dos $30^{\circ} \mathrm{C}$ por grande parte do ano, atingindo muitas vezes, entre 35 e $38^{\circ} \mathrm{C}$. O Brasil insere-se na faixa do planeta considerado quente (Baccari Junior, 2001). O ambiente térmico representa, portanto, fator de restrição para a eficiência máxima de produção de bovinos leiteiros, principalmente nos sistemas intensivos. A produção de leite em ambiente tropical pode ser melhorada a partir do uso de tecnologias que garantam o manejo adequado do rebanho.

O estresse térmico, além de acarretar mudanças nas reações fisiológicas e comportamentais, também desencadeia alterações agudas e crônicas nas concentrações plasmáticas de cortisol e hormônios tiroideanos $\left(\mathrm{T}_{3}\right.$ e $\left.\mathrm{T}_{4}\right)$. Segundo Smith (1993) as concentrações normais de cortisol para bovinos encontram-se em torno de $0,35 \mu \mathrm{g} \mathrm{dL}{ }^{-1}$, enquanto que as concentrações de triiodotironina $\left(\mathrm{T}_{3}\right)$ variam de 41 a $70 \mu \mathrm{g} \mathrm{dL}^{-1}$ e da tiroxina $\left(\mathrm{T}_{4}\right) \mathrm{de}$ 3,6 a $8,9 \mu \mathrm{g} \mathrm{mL}^{-1}$. A atividade tireoideana se reduz em resposta ao incremento do estresse calórico ao mesmo tempo em que se elevam os níveis de cortisol (Starling \& Silva, 1998)

Christison \& Johnson (1972) encontraram concentrações plasmáticas significativamente mais baixas de cortisol, quando comparadas ao grupo de vacas em ambientes termoneutro, enquanto que Wise et al. (1988) encontraram concentrações plasmáticas de cortisol mais elevadas em vacas em condições de estresse térmico comparadas às mantidas sob refrigeração.

A redução de ingestão de alimentos resulta em um decréscimo da produção de calor e é uma estratégia usada pelo animal para manter a sua temperatura corporal constante. Head (1989) afirmou que o estresse térmico causou decréscimos na produção e no teor de constituintes do leite, como a gordura, proteína, ácido cítrico, cálcio e potássio. Em conseqüência de sua ação sobre o consumo, o estresse calórico tem efeitos marcantes sobre o metabolismo da glândula mamária e da composição do leite.

Durante o estresse crônico com temperatura ambiente acima de $21^{\circ} \mathrm{C}$, vacas em lactação apresentaram mudanças fisiológicas na produção quanto a composição do leite, bem como flutuações nos níveis de cortisol (Giesecke, 1985). Alguns efeitos resultam de alterações (redução) na síntese, absorção e mobilização dos metabólitos (glicose, ácidos graxos voláteis, lipídeos, aminoácidos, etc.) a partir do trato digestivo, fígado e tecido adiposo, e sua utilização pela glândula mamária (Head, 1989). Com a temperatura em torno de $36^{\circ} \mathrm{C}$, segundo Rodriguez et al. (1985), há um decréscimo de 0,54 e $0,44 \%$ na percentagem de gordura e proteína do leite, respectivamente.

O resfriamento evaporativo dos abrigos para animais, particularmente gado leiteiro, tem-se expandido rapidamente em climas quentes, devido à sua relativa simplicidade técnica e praticidade em termos de uma relação benefício/custo favorável, fatores que têm resultado na sua aceitação por muitos criadores.

Igono et al. (1985) utilizaram aspersão de água em vacas holandesas durante o verão no estado de Columbia (EUA), e encontraram acréscimos de $0,7 \mathrm{~kg}$ de leite quando as vacas foram comparadas com as sem aspersão. Du Preez (2000) encontrou diminuição da gordura e proteína do leite de vacas sob condição de estresse térmico, quando comparadas com vacas submetidas à ventilação e aspersão, enquanto Arcaro Júnior et al. (2001) não encontrou diferença na porcentagem de gordura do leite de vaca sem lactação que permaneceram por 15 min em sala de espera climatizada.

Arcaro Júnior et al. (2000) trabalhando com 18 vacas em piquetes, recebendo três tratamentos (sombra artificial com sombrite, sombrite mais ventilação e sombrite mais ventilação e aspersão), encontraram aumentos na produção de leite de $18,20,19,15$ e $20,53 \mathrm{~kg}$, respectivamente.

O trabalho teve por objetivo avaliar a eficiência da climatização do ambiente da sala de espera (ventilação ou ventilação mais aspersão) sobre a produção e composição do leite (gordura, proteína, lactose) e os níveis plasmáticos de cortisol, $\mathrm{T}_{3}$ e $\mathrm{T}_{4}$.

\section{MATERIAL E MÉTODOS}

O presente ensaio foi conduzido na Estação Experimental de Zootecnia, localizada no município de Nova Odessa, SP, com Latitude $22^{\circ} 42^{\prime}$ S e Longitude $47^{\circ} 18^{\prime}$ W e altitude de $550 \mathrm{~m}$. Foram utilizadas 21 vacas entre a terceira e a quarta lactação, com peso médio de $600 \mathrm{~kg}$, após o pico de lactação (60 dias), com produção média de $21 \mathrm{~kg} \mathrm{~d}^{-1}$, que foram distribuídas aleatoriamente dentro dos seguintes tratamentos:

- sala de espera sem climatização (Controle) - CONTR

- sala de espera com ventilação - V

- sala de espera com ventilação e aspersão - VA

Os animais receberam, como alimentação, silagem de sorgo, farelo de soja, grãos de milho moído, caroço de algodão e minerais, fornecidos na forma de dieta completa e de maneira a atender as exigências nutricionais para a manutenção e produção de leite, de acordo com o NRC (1989). Os animais foram ordenhados e suas produções registradas diariamente, três vezes ao dia (7, 14 e 21 h). Amostras semanais para a determinação dos componentes do leite (gordura, proteína e lactose) foram colhidas semanalmente, sendo a análise da composição do leite efetuada através do método espectrométrico por absorção no infravermelho em equipamento Bentley 2000.

Os equipamentos de climatização da sala de espera foram acionados após a ordenha dos animais-controle, somente quando a temperatura atingia $25^{\circ} \mathrm{C}$. Os animais do tratamento $\mathrm{V}$ permaneceram na sala de espera por $30 \mathrm{~min}$ com os ventiladores ligados, sendo depois ordenhados. Posteriormente, foram ligados os ventiladores e os aspersores para o lote correspondente ao tratamento VA, e estes também permaneciam por 30 min para depois serem ordenhados. O registro da temperatura, umidade relativa do ar e temperatura de globo negro foi feito com sensores higrotérmicos (psicrômetros) e termômetro de globo negro, instalados dentro da sala de espera. 
Para a determinação plasmática dos hormônios cortisol, triiodotironina $\left(\mathrm{T}_{3}\right)$ e tiroxina $\left(\mathrm{T}_{4}\right)$ foram colhidas, semanalmente, amostras de sangue de cada vaca, da veia coccígea. As amostras foram centrifugadas para a obtenção do soro e acondicionadas em freezer até o momento da análise. As concentrações de $T_{3}$, $\mathrm{T}_{4}$ e cortisol foram determinadas por radioimunoensaio (RIE) em fase sólida.

As análises dos dados foram feitas pelo programa computacional SAS, procedimento Anova (SAS, 1990).

\section{RESULTADOS E DISCUSSÃO}

Os valores médios da temperatura de bulbo seco (TBS), umidade relativa do ar (UR) e temperatura de globo negro (TGN) na sala de espera, antes e após a aplicação dos tratamentos (CONTR, V e VA) às $14 \mathrm{~h}$, encontram-se na Tabela 1 . A temperatura média de bulbo seco permaneceu abaixo dos $25^{\circ} \mathrm{C}$ nos horários das $8 \mathrm{e} 21 \mathrm{~h}$, atingindo os $26,12^{\circ} \mathrm{C}$ às $14 \mathrm{~h}$, quando então os equipamentos foram acionados. Os valores de TBS às 8 e $21 \mathrm{~h}$ permaneceram dentro da zona de conforto térmico, onde o gasto de energia para mantença dos animais é mínimo, a variação da temperatura corporal e da freqüência respiratória é normal, e a produção é ótima (Baccari Jr., 1998). Existe muita discussão sobre os limites de zona de conforto térmico; segundo Nääs (1989), a faixa de conforto térmico para vacas em lactação recomendada estaria entre 4 e $24{ }^{\circ} \mathrm{C}$. A TBS e TGN diminuíram significativamente com os tratamentos, sendo que o tratamento VA foi mais eficiente na diminuição da temperatura ambiente da sala de ordenha para níveis de conforto térmico dos animais. Arcaro Júnior et al. (2000), comparando três sistemas de climatização (sombra, sombra com ventilação e sombra com ventilação e aspersão) encontraram reduções na TBS de 2,9 e $3,9{ }^{\circ} \mathrm{C}(10$ e $12 \%)$ respectivamente, em relação ao tratamento com sombra. A umidade relativa do ar, como esperado, aumentou significativamente para o tratamento VA. O aumento da umidade relativa do ar associado a temperaturas elevadas são os principais elementos estressores. Segundo Jonhson \& Vanjonack (1976), a umidade relativa do ar de $80 \%$ e temperatura de $34^{\circ} \mathrm{C}$ levou a queda na produção de leite de $40 \%$. A utilização de sistema de aspersão em épocas de verão chuvoso, onde existe uma elevação da umidade relativa do ar, pode causar uma umidade excessiva nas instalações e prejudicar a eficiência do sistema.

Os valores médios da produção e composição do leite dos animais nos diferentes tratamentos estão sumarizados na Tabela 2.

Não houve diferença entre os tratamentos em relação à produção de leite e produção corrigida a 4\%, cujos dados estão de acordo com os observados por Arcaro Júnior et al. (2001), que não encontraram diferença na produção de leite em vacas que permaneceram em sala de espera climatizada por $15 \mathrm{~min}$.

Neste experimento, o valor médio da temperatura ambiente no horário das $14 \mathrm{~h}$ foi de $28,8^{\circ} \mathrm{C}$, entretanto, não se verificou efeito dos tratamentos para a produção de leite, estando de acordo com as conclusões de Rodriguez et al. (1985) e Yousef \& Johnson (1985) de que uma queda brusca na produção ocorre quando a temperatura ambiente excede $29^{\circ} \mathrm{C}$, ou a falta de resposta pode ser atribuída ao curto espaço de tempo em que os animais foram expostos ao ambiente climatizado. Estes resultados diferem dos encontrados por Arcaro Júnior et al. (2000) que, trabalhando com 18 vacas em lactação alojadas em piquetes providos de sombras artificiais produzidas por tela de propileno $80 \%$, sombras idênticas às anteriores, acrescidas de ventilação forçada e sombra combinando ventilação e aspersão, encontraram diferenças significativas nos valores da produção de leite, com o tratamento sombra ventilação e aspersão apresentando a melhor média $(20,53 \mathrm{~kg})$, seguido de sombra mais ventilação $(19,19 \mathrm{~kg})$ e sombra $(18,20 \mathrm{~kg})$.

Pode-se observar diferenças significativas entre os tratamentos para os valores de gordura e sólidos totais. O leite dos animais que receberam apenas ventilação apresentou maior teor de gordura em relação aos demais tratamentos (Tabela 2).

Tabela 1. Valores médios* da temperatura de bulbo seco (TBS), umidade relativa do ar (UR) e temperatura do bulbo negro (TGN), verificados na sala de espera antes e depois da aplicação dos tratamentos

\begin{tabular}{|c|c|c|c|c|c|c|}
\hline \multirow{3}{*}{ Tratamentos } & \multicolumn{6}{|c|}{ Variáveis Climáticas } \\
\hline & \multicolumn{2}{|c|}{$\operatorname{TBS}\left({ }^{\circ} \mathrm{C}\right)$} & \multicolumn{2}{|c|}{ UR (\%) } & \multicolumn{2}{|c|}{$\mathrm{TGN}\left({ }^{\circ} \mathrm{C}\right)$} \\
\hline & Antes & Depois & Antes & Depois & Antes & Depois \\
\hline Ventilação & $27,53 \mathrm{~b}$ & $29,34 \mathrm{a}$ & $45,93 \mathrm{a}$ & $38,92 \mathrm{c}$ & $27,66 \mathrm{~b}$ & $29,00 \mathrm{a}$ \\
\hline Ventilação + Aspersão & $29,34 \mathrm{a}$ & $22,94 \mathrm{c}$ & $38,95 \mathrm{~b}$ & $79,78 \mathrm{a}$ & $29,01 \mathrm{a}$ & $22,49 c$ \\
\hline
\end{tabular}

"Médias seguidas da mesma letra nas linhas não diferem pelo teste de Duncan ao nível de $5 \%$ de probabilidade

Tabela 2. Valores médios* da produção de leite $\left(\mathrm{kg} \mathrm{d}^{-1}\right)$, porcentagem de gordura, lactose, proteína e sólidos totais, referentes aos tratamentos

\begin{tabular}{|c|c|c|c|c|c|c|}
\hline \multirow{3}{*}{ Tratamentos } & \multicolumn{6}{|c|}{ Produção e Composição do Leite } \\
\hline & $\begin{array}{l}\text { Prod. } \\
\text { Leite }\end{array}$ & $\begin{array}{l}\text { Prod Leite } \\
\text { Corrig 4\% }\end{array}$ & Gordura & Lactose & Proteína & $\begin{array}{l}\text { Sólidos } \\
\text { Totais }\end{array}$ \\
\hline & \multicolumn{3}{|c|}{$\mathrm{kg}$} & \multicolumn{3}{|c|}{$\%$} \\
\hline Controle & $21,17 \mathrm{a}$ & $20,60 \mathrm{a}$ & $3,81 \mathrm{~b}$ & $4,60 \mathrm{a}$ & $3,16 \mathrm{a}$ & $11,76 \mathrm{a}$ \\
\hline Ventilação & $21,73 \mathrm{a}$ & $22,52 \mathrm{a}$ & $4,20 \mathrm{a}$ & $4,74 \mathrm{a}$ & $3,39 \mathrm{a}$ & $12,50 \mathrm{a}$ \\
\hline Vent. + aspersão & $21,90 \mathrm{a}$ & $21,10 \mathrm{a}$ & $3,73 \mathrm{~b}$ & $4,64 \mathrm{a}$ & $3,15 \mathrm{a}$ & $11,57 \mathrm{a}$ \\
\hline C.V. $(\%)$. & 18,84 & 10,92 & 7,29 & 7,63 & 10,43 & 6,57 \\
\hline
\end{tabular}

"Médias seguidas da mesma letra nas colunas não diferem pelo teste de Duncan ao nível de $5 \%$ de probabilidade 
Segundo Head (1989) e Rodriguez et al. (1985), altas temperaturas provocam queda na produção de gordura e nos componentes do leite.

O estresse crônico, segundo Giesecke (1985), causa flutuações tanto na produção de leite como na sua composição. De acordo com Du Preez (2000), a porcentagem de gordura e proteína em animais sob estresse térmico é menor que em animais resfriados (4,2 e 3,5\%, respectivamente).

A concentração média plasmática de cortisol aumentou significativamente nas vacas-controle, sendo menor para as vacas submetidas à ventilação, seguidas das que receberam ventilação mais aspersão (Tabela 3). Apesar dos valores de triiodotironona $\left(\mathrm{T}_{3}\right)$ e tiroxina $\left(\mathrm{T}_{4}\right)$ diferirem significativamente neste experimento, eles se situam dentro da faixa de normalidade que, segundo Smith (1993) estariam para o cortisol em torno de $0,35 \mu \mathrm{g} \mathrm{dL}{ }^{-1}$; para o $\mathrm{T}_{3}$ os valores variam entre 41 a $170 \mu \mathrm{g} \mathrm{dL}^{-1}$ e para o $\mathrm{T}_{4}$ de 3,6 a $8,9 \mu \mathrm{g} \mathrm{mL}^{-1}$.

Tabela 3. Valores médios* referentes aos níveis hormonais de cortisol, Tiroxina $\left(\mathrm{T}_{4}\right)$ e Triiodotironina $\left(\mathrm{T}_{3}\right)$ aos três grupos experimentais

\begin{tabular}{lccc}
\multirow{2}{*}{\multicolumn{1}{c}{ Tratamentos }} & \multicolumn{3}{c}{ Parâmetros $\left(\mu \mathrm{gL}^{-1}\right)$} \\
\cline { 2 - 4 } & Cortisol & $\mathrm{T}_{3}$ & $\mathrm{~T}_{4}$ \\
\hline Controle & $1,000 \mathrm{a}$ & $84,04 \mathrm{a}$ & $4,23 \mathrm{a}$ \\
Ventilação & $0,928 \mathrm{~b}$ & $85,42 \mathrm{~b}$ & $3,94 \mathrm{~b}$ \\
Ventilação + aspersão & $0,705 \mathrm{c}$ & $77,55 \mathrm{c}$ & $3,65 \mathrm{c}$ \\
C.V. (\%). & 20,427 & 13,161 & 10,319 \\
\hline
\end{tabular}

Médias seguidas da mesma letra nas colunas não diferem pelo teste de Duncan ao nível de $5 \%$ de probabilidade

Em relação ao cortisol, os resultados concordam com Wise et al. (1988) e Starling \& Silva (1998) que encontraram níveis elevados de cortisol plasmático sob estresse térmico quando comparado com vacas mantidas à temperatura de $22{ }^{\circ} \mathrm{C}$. Du Preez (2000) encontrou concentração média plasmática de cortisol de 59,2 $\mathrm{n} \mathrm{mol}^{-1}$ nas vacas submetidas a estresse e 45,4 $\mathrm{n}$ mol $^{-1}$ nas vacas mantidas sob refrigeração. Segundo Christison \& Johnson (1972) a curta exposição ao estresse térmico aumenta os níveis de cortisol, enquanto a longa exposição os diminui e, portanto, a concentração plasmática de cortisol diminui em animais sob estresse crônico quando comparado com os níveis de cortisol de animais mantidos sob termoneutralidade. A redução dos níveis dos glicocorticóides pode ocorrer também durante a aclimatação térmica, como mecanismo benéfico na redução da produção de calor dos animais.

Collier et al. (1981) também encontraram níveis elevados de cortisol durante o estresse agudo. Há evidências de que o cortisol, juntamente com outros hormônios, influencia o metabolismo da glândula mamária.

O presente trabalho sugere que as altas concentrações deste hormônio catabólico podem ter sido produzidas pelos animais sob condições de estresse em detrimento de manter a produção de leite, o que concorda com Wise et al. (1988).

Em trabalho desenvolvido com ovinos da raça Corriedale, verificou-se que, durante a fase inicial do estresse pelo calor, os níveis hormonais de $\mathrm{T}_{3}$ e $\mathrm{T}_{4}$ aumentaram, sugerindo então que o estresse pelo calor produz mudanças nos níveis de $\mathrm{T}_{3} \mathrm{e}$, especialmente, $\mathrm{T}_{4}$, ambos em magnitude e direção, de acordo com a duração da exposição (Nascimento \& Silva, 2000).

\section{CONCLUSÕES}

1. O tratamento ventilação mais aspersão proporcionou maiores reduções nas variáveis climáticas, resultando em melhores condições de conforto térmico.

2. O tempo de permanência dos animais na sala de ordenha climatizada de 30 min não proporcionou aumento na produção de leite.

3. As concentrações plasmáticas do cortisol elevadas caracterizaram a condição de estresse dos animais, em todos os tratamentos.

\section{LITERATURA CITADA}

Arcaro Júnior, I.; Arcaro, J. R. P.; Pozzi, C. R.; Matarazzo, S. V.; Fagundes, H. Efeitos da climatização em sala de espera sobre a produção e composição do leite e os parâmetros fisiológicos de vacas em lactação. In: Congresso Brasileiro de Biometeorologia, 3, 2001, Maringá. Anais... Maringá, 2001. CD-Rom

Arcaro Júnior, I.; Nääs, I.A.; Arcaro, J.R.P. Dairy response for shade associated to cooling equipment. Transactions of the ASAE, St. Joseph, p.1-8, 2000.

Baccari Júnior, F. Adaptação de manejo na produção de leite em clima quente. In: I Simpósio Brasileiro de Ambiência na Produção de Leite, 1998, Piracicaba. Anais...Piracicaba: FEALQ. 1998. p. 24-67.

Baccari Júnior, F. Manejo ambiental da vaca leiteira em climas quentes. 1 ed. Londrina: UEL Editora, 2001.141p.

Christison, G.I.; Johnson, H.D. Cortisol turnover in heat-stressed cows. Journal of Animal Science, Champaign, v.35, n.5, p.1005-1010, 1972.

Collier, R.J.; Eley, R.M.; Sharma, A.K.; Pereira, R.M.; Bufington, D.E. Shade management in a subtropical environment for milk yield and composition in Holtein and Jersey cows. Journal of Dairy Science, Lancaster, v. 64, p. 844-849, 1981.

Du Preez, J.H. Parameters for the determination and evaluation of heat stress in dairy cattle in South Africa. Journal of Veterinary Research, v. 67,p. 263-271, 2000.

Giesecke, W.H. The effect of stress on udder health of dairy cows. Onderstepoort Journal of Veterinary Research, v.52, p.175-193, 1985 .

Head, H.H. The strategic use of the physiological potential of the dairy cow. In: Simpósio Leite nos Trópicos: novas estratégias de produção, 1989, Botucatu. Anais... Botucatu, 1989. p. 38-89.

Igono, M.O., Stevens, B.J.; Shanklin, M.D.; Johson, H.D. Spray cooling effects on milk production, milk and retal temperature of cows during a moderate temperature summer season. Journal of Dairy Science, Lancaster, v. 68, p. 979-85, 1985.

Johnson, H.D.; Vanjonack, W.J. Effects of environmental and other stressors on blood hormone patterns in lactating animals. Journal Dairy Science, Lancaster, v.59, p.1603-17,1976.

Nääs, I.A. Principios de conforto térmico na produção animal, São Paulo: Ícone Editora Ltda, 1989. 183p. 
Nascimento, M.; Silva, R.G. Efeito de fatores ambientais sobre os níveis de 3, 5, 3, - triiodotironina $\left(\mathrm{T}_{3}\right)$ e tiroxina $\left(\mathrm{T}_{4}\right)$ em ovinos corriedale. Botucatu. UNESP, 2000. Tese Douto-rado

NRC - Nutrient requirements of dairy cattle. Washington, D.C.: National Academy of Science, National Academy Press, 1989. 202p.

Rodriguez, L.A.; Mckonnen, G.; Wilcox, C.J.; Martin, F.G.; Krienke, W.A. Effects of relative humidity, maximum and minimum temperature, pregnancy and stage of lactation milk composition and yeld. Journal of Dairy Science, Lancaster, v.68, p.973-978. 1985.

SAS - Stastistical Analisis System. User' Guide: Statistics (Release 6.03). SAS Inst., Inc., 1990.
Smith, B.P. Tratado de medicina veterinária interna de grandes animais. 1. ed. São Paulo: Manole Ed. Ltda, 1993. 900p.

Starling, J.M.C.; Silva, R. G. Variação nos níveis de cortisol, T3 e T4 de ovinos em ambiente tropical. In: Congresso Brasileiro de Biometeorologia, 2, 1998, Goiânia. Anais... Goiânia, 1998. p.367-375.

Wise, M.E.; Armstrong, D.V.; Huber, J.T.; Hunter, R.; Wiersma, F. Hormonal alterations in the lactating dairy cow in response to thermal stress. Journal of Dairy Science, Lancaster, v.71, p.2480-2485, 1988.

Yousef, H.M.K.; Jonhson, H.D. Endocrine system and thermal environment, in stress physiology in livestock. Basic principles, 1. ed. Florida: Boca Raton, 1985. 139p. 